\title{
FIRST SPECTROSCOPIC IDENTIFICATION OF MASSIVE YOUNG STELLAR OBJECTS IN THE GALACTIC CENTER
}

\author{
Deokkeun An ${ }^{1}$, Solange V. Ramírez ${ }^{2}$, Kris Sellgren ${ }^{3}$, Richard G. Arendt ${ }^{4}, 10$, A. C. Adwin Boogert ${ }^{1}$, \\ Mathias Schultheis ${ }^{5,6}$, Susan R. Stolovy ${ }^{7}$, Angela S. Cotera ${ }^{8}$, Thomas P. Robitaille ${ }^{9}, 11$, And Howard A. Smith ${ }^{9}$ \\ ${ }^{1}$ Infrared Processing and Analysis Center, California Institute of Technology, Mail Stop 100-22, Pasadena, CA 91125, USA; deokkeun@ipac.caltech.edu \\ ${ }^{2}$ NASA Exoplanet Science Institute, California Institute of Technology, Mail Stop 100-22, Pasadena, CA 91125, USA \\ ${ }^{3}$ Department of Astronomy, Ohio State University, 140 West 18th Avenue, Columbus, OH 43210, USA; sellgren@astronomy.ohio-state.edu \\ ${ }^{4}$ CRESST/UMBC/GSFC, Code 665, NASA/Goddard Space Flight Center, 8800 Greenbelt Road, Greenbelt, MD 20771, USA \\ ${ }^{5}$ Observatoire de Besançon, 41 bis, avenue de l'Observatoire, F-25000 Besançon, France \\ ${ }^{6}$ Institut d'Astrophysique de Paris, CNRS, 98 bis Bd Arago, F-75014 Paris, France \\ ${ }^{7}$ Spitzer Science Center, California Institute of Technology, Mail Code 220-6, 1200 East California Boulevard, Pasadena, CA 91125, USA \\ ${ }^{8}$ SETI Institute, 515 North Whisman Road, Mountain View, CA 94043, USA \\ ${ }^{9}$ Harvard-Smithsonian Center for Astrophysics, 60 Garden Street, Cambridge, MA 02138, USA \\ Received 2009 June 9; accepted 2009 July 27; published 2009 August 21
}

\begin{abstract}
We report the detection of several molecular gas-phase and ice absorption features in three photometrically selected young stellar object (YSO) candidates in the central $280 \mathrm{pc}$ of the Milky Way. Our spectra, obtained with the Infrared Spectrograph (IRS) onboard the Spitzer Space Telescope, reveal gas-phase absorption from $\mathrm{CO}_{2}(15.0 \mu \mathrm{m}), \mathrm{C}_{2} \mathrm{H}_{2}(13.7 \mu \mathrm{m})$, and $\mathrm{HCN}(14.0 \mu \mathrm{m})$. We attribute this absorption to warm, dense gas in massive YSOs. We also detect strong and broad $15 \mu \mathrm{m} \mathrm{CO}$ ice absorption features, with a remarkable double-peaked structure. The prominent long-wavelength peak is due to $\mathrm{CH}_{3} \mathrm{OH}$-rich ice grains, and is similar to those found in other known massive YSOs. Our IRS observations demonstrate the youth of these objects, and provide the first spectroscopic identification of massive YSOs in the Galactic Center.
\end{abstract}

Key words: infrared: ISM - ISM: molecules - stars: formation

\section{INTRODUCTION}

The Central Molecular Zone (CMZ) is the innermost $\sim 200 \mathrm{pc}$ region of the Milky Way Galaxy. It is a giant molecular cloud complex delineated by a gradient in the CO column density and temperature. The CMZ contains $\sim 10 \%$ of the Galaxy's molecular gas, and produces $5 \%-10 \%$ of its infrared and Lyman continuum luminosities (see a review by Morris \& Serabyn 1996, and references therein).

Evidence is mounting that conditions for star formation in the $\mathrm{CMZ}$ are significantly different from those in the Galactic disk. The gas pressure and temperature are higher in the CMZ than in the average disk, conditions that favor a larger Jeans mass for star formation and an initial mass function biased toward more massive stars. Furthermore, the presence of strong magnetic fields, tidal shear, and turbulence challenges the standard paradigm of slow gravitational collapse of molecular cloud cores.

The CMZ provides several signposts of in situ star formation, such as $\mathrm{H}_{2} \mathrm{O}$ masers, (ultra-)compact $\mathrm{H}$ II regions, young $\mathrm{OB}$ stars, and young supernova remnants. However, young stellar objects (YSOs or protostars), which are the direct tracers of current star formation, have so far eluded detection in the CMZ. They have been inferred to be present based on infrared photometry (e.g., Felli et al. 2002; Schuller et al. 2006; YusefZadeh et al. 2009), but spectroscopic observations are required to confirm their status as a YSO. This is because evolved stars can look like YSOs in broadband photometry, if they are heavily dust attenuated (e.g., Schultheis et al. 2003), a problem toward the Galactic Center (GC), where $A_{V} \approx 30$.

\footnotetext{
${ }^{10}$ Science Systems and Applications, Inc.

${ }^{11}$ Spitzer Postdoctoral Fellow.
}

In this Letter, we present spectroscopic follow-up observations of YSO candidates in the CMZ, using the Infrared Spectrograph (IRS; Houck et al. 2004) onboard the Spitzer Space Telescope (Werner et al. 2004). Massive YSO candidates were photometrically selected from the Point Source Catalog (Ramírez et al. 2008), which was extracted from images of the CMZ (Stolovy et al. 2006) made using the Infrared Array Camera (IRAC; Fazio et al. 2004). This high sensitivity and high spatial resolution image has led to a better identification of YSO candidates and their follow-up spectroscopic observations.

\section{PHOTOMETRIC SAMPLE SELECTION}

The IRAC Point Source Catalog (Ramírez et al. 2008) contains photometry for more than a million point sources in the entire CMZ $\left(2^{\circ} \times 1.4\right.$ or $\left.280 \times 200 \mathrm{pc}\right)$ in four channels (3.6 $\mu \mathrm{m}, 4.5 \mu \mathrm{m}, 5.8 \mu \mathrm{m}$, and $8.0 \mu \mathrm{m})$. Initially, we selected point sources with [3.6] $-[8.0] \geqslant 2.0$, corresponding to YSOs with $M_{*} \gtrsim 2.5 M_{\odot}$ (Whitney et al. 2003, 2004). We further confined the sample to those within $|b|<15^{\prime}$, resulting in 1207 objects. When we had photometric measurements in at least five bandpasses from IRAC, Two Micron All Sky Survey (2MASS; $J H K_{s}$; Skrutskie et al. 2006), and/or ISOGAL (7 $\mu \mathrm{m}$ and $15 \mu \mathrm{m}$; Omont et al. 2003), we selected YSO candidates by comparing the observed spectral energy distribution (SED) with YSO models (Robitaille et al. 2006) using an SED fitting tool by Robitaille et al. (2007). Otherwise, we applied additional color constraints from Whitney et al. $(2004,[3.6]-[4.5] \geqslant 0.5$, $[4.5]-[5.8] \geqslant 0.5$, and $[5.8]-[8.0] \geqslant 1.0)$ to identify YSO candidates. SED fitting and color selection narrowed down our sample to about 200 objects.

Then, we carefully inspected IRAC three-color images to select objects that are distinct within the IRS slit entrances against 
the crowded stellar field and bright local background. Finally, a literature search was carried out for the selected objects, and one Wolf-Rayet star and four OH/IR stars were discarded. Our final sample is composed of 107 objects, among which 25 were previously known YSO candidates from ISOGAL (Felli et al. 2002).

\section{IRS OBSERVATIONS AND DATA REDUCTION}

We obtained spectroscopic data for 107 YSO candidates using the four IRS modules in 2008 May and October. We observed each target in IRS staring mode with four exposures per source (two cycles). Exposure times were 6-120 s in SH (short-high; short wavelength, high resolution), 6-60 s in LH (long-high), 6-14 s in SL (short-low), and $6 \mathrm{~s}$ in LL (long low) modules, depending on the source's brightness, to achieve a signal-tonoise ratio $(\mathrm{S} / \mathrm{N})$ of at least 50 in $\mathrm{SH}$ and $\mathrm{SL}$, and a minimum $\mathrm{S} / \mathrm{N}$ of $10 \mathrm{in} \mathrm{LH}$ and LL. We reduced the IRS spectra from the basic calibrated data (BCD) products version S17.2.0 and S18.1.0, using the SSC software packages IRSCLEAN (to correct for bad pixel values) and SPICE (to extract spectra).

Because the GC exhibits strong, spatially variable background, we observed multiple off-source measurements (one cycle, $1 \times 1$ mapping mode) to derive backgrounds near each of our YSO candidates in the four IRS modules. The on-source and the off-source observations were taken consecutively to minimize zodiacal light and instrumental variations. For the high-resolution observations, we observed and extracted four background positions ( $\sim \pm 1^{\prime}$ offsets in either R.A. or decl.). For the low-resolution observations, we took spectra from two background positions at $\sim \pm 1^{\prime}$ away in the direction perpendicular to the slit, and extracted two additional background spectra at positions along the on-source slit. In all of the four different IRS modules, we tried to extract the background spectra at the same position as much as possible, to minimize the flux difference from different modules.

We made an interpolation of a plane in three-dimensional space (positions on the IRAC map and wavelength) to obtain a background spectrum at the source position. We estimated an error in each source's background from the dispersion of four different background spectra, constructed from alternate sets of three out of the four background pointings.

A complete analysis of spectra for all of our 107 YSO candidates will be presented elsewhere (D. An et al. 2009, in preparation). For the current analysis, we selected three targets (Table 1) from among those showing characteristic spectral features of massive YSOs, which include gaseous molecular absorptions from $\mathrm{C}_{2} \mathrm{H}_{2}, \mathrm{HCN}, \mathrm{CO}_{2}$ (e.g., Lahuis \& van Dishoeck 2000; Boonman et al. 2003; Knez et al. 2009), and a solid-phase absorption from the $\mathrm{CO}_{2}$ ice bending mode (e.g., Gerakines et al. 1999).

Both SSTGC 797384 and SSTGC 803187 are associated with a relatively weak radio continuum source (SGR B2(P) and SGR B2(R), respectively; Mehringer et al. 1993). They are on the outskirts of the Sgr B2 molecular cloud $(\sim 2-$ $4 \mathrm{pc}$ from the well-studied radio source SGR B2(M)), which is one of the most active complexes of compact $\mathrm{H}$ II regions in the Galaxy (e.g., Mehringer 1995). Mehringer et al. (1993) derived zero-age main-sequence spectral types of B0 and 06.5 for these compact $\mathrm{H}$ II regions, respectively, from the number of ionizing photons. SSTGC 524665 does not have radio continuum emission associated with it. However, it is coincident with an $\mathrm{H}_{2} \mathrm{O}$ maser (Forster \& Caswell 1989), and is adjacent to a region of $4.5 \mu \mathrm{m}$ excess emission (Yusef-Zadeh et al. 2009),

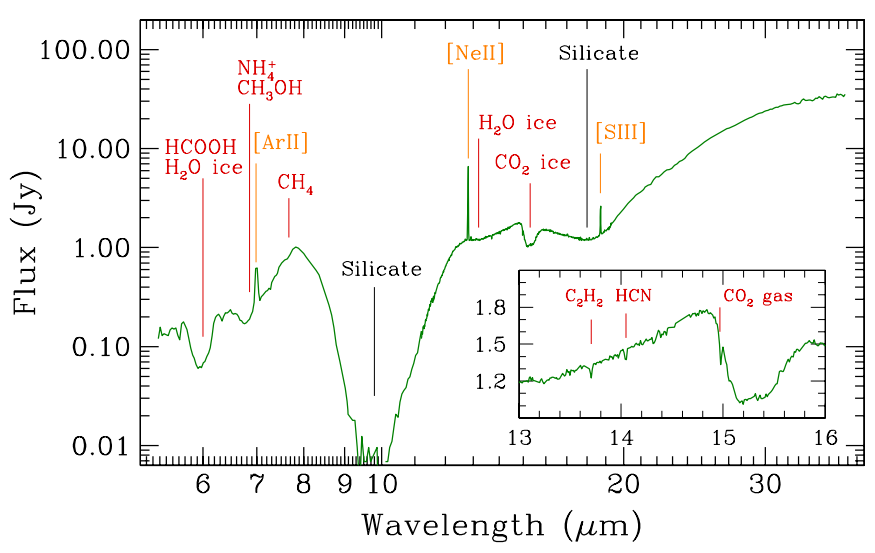

Figure 1. Composite IRS spectrum of SSTGC 797384. The spectrum is from $\mathrm{SL}$ at $\lambda \leqslant 11.2 \mu \mathrm{m}, \mathrm{SH}$ at $11.2 \mu \mathrm{m} \leqslant \lambda \leqslant 19.3 \mu \mathrm{m}$, and LL at $\lambda \geqslant 19.3 \mu \mathrm{m}$. This composite spectrum is characterized by an extremely red spectral energy distribution, strong and deep silicate absorption, and several molecular gas- and solid-phase absorptions.

possibly tracing shocked molecular outflows (e.g., Smith et al. 2006).

For SSTGC 803187, we used a nonstandard extraction aperture in SL, because of a nearby source $\left(\approx 7^{\prime \prime}\right.$ south of the target) along the slit. We followed the prescription on the IRS data reduction Web site ${ }^{12}$ to calibrate the flux. We trimmed the end of the orders to remove the noisy part of spectra, and spectra from different orders in high-resolution modules were averaged using a linear ramp. After background subtraction, the SH and LH spectra were scaled down in flux to LL over the common wavelength interval for SSTGC 797384 and SSTGC 803187. The SL spectra were then scaled to SH. For these sources, we assumed that the flux mismatch is due to narrower slit entrances in SH and SL. For SSTGC 524665, we used the SL as a basis for the scaling, because our observations in LL and LH were contaminated by extended emission from a nearby $\left(\approx 10^{\prime \prime}\right.$ southwest of the target) bright source on the $24 \mu \mathrm{m}$ image using the Multiband Imaging Photometer for Spitzer (MIPS) (Carey et al. 2009; Yusef-Zadeh et al. 2009). The background for this target is likely to be oversubtracted, because the target lies on a dark cloud with high extinction, while background spectra were taken at brighter spots. The potential problem of the background subtraction results in $\mathrm{H}_{2}$ lines (arising from the surrounding sky) appearing in absorption in SSTGC 524665. In the following initial analysis, we did not use LH data for all targets, but focused on the spectral features in other modules.

\section{ANALYSIS AND RESULTS}

Figure 1 displays background-subtracted spectra of SSTGC 797384 , in SL $(\lambda \leqslant 11.2 \mu \mathrm{m}), \mathrm{SH}(11.2 \mu \mathrm{m} \leqslant \lambda \leqslant 19.3 \mu \mathrm{m})$, and $\operatorname{LL}(\lambda \geqslant 19.3 \mu \mathrm{m})$. The observed spectrum is characterized by an extremely red $\operatorname{SED}\left[\alpha \equiv d \log \left(\lambda F_{\lambda}\right) / d \log (\lambda) \approx 2\right]$, strong and deep silicate absorptions at $9.7 \mu \mathrm{m}$ and $18 \mu \mathrm{m}$, ice absorption features at $6 \mu \mathrm{m}, 6.85 \mu \mathrm{m}, 13 \mu \mathrm{m}$, and $15.2 \mu \mathrm{m}$. Although the presence of forbidden lines indicates that these objects are likely associated with an (ultra-)compact $\mathrm{H}$ II region, it could be also due to undersubtracted emissions from the background.

Figure 2 shows gas-phase molecular absorptions at $13.71 \mu \mathrm{m}$ $\left(\mathrm{C}_{2} \mathrm{H}_{2} v_{5}=1-0\right), 14.05 \mu \mathrm{m}\left(\mathrm{HCN} v_{2}=1-0\right)$, and $14.97 \mu \mathrm{m}$ $\left(\mathrm{CO}_{2} v_{2}=1-0\right)$, detected in three YSO candidates. To derive

\footnotetext{
12 See http://ssc.spitzer.caltech.edu/IRS/calib.
} 


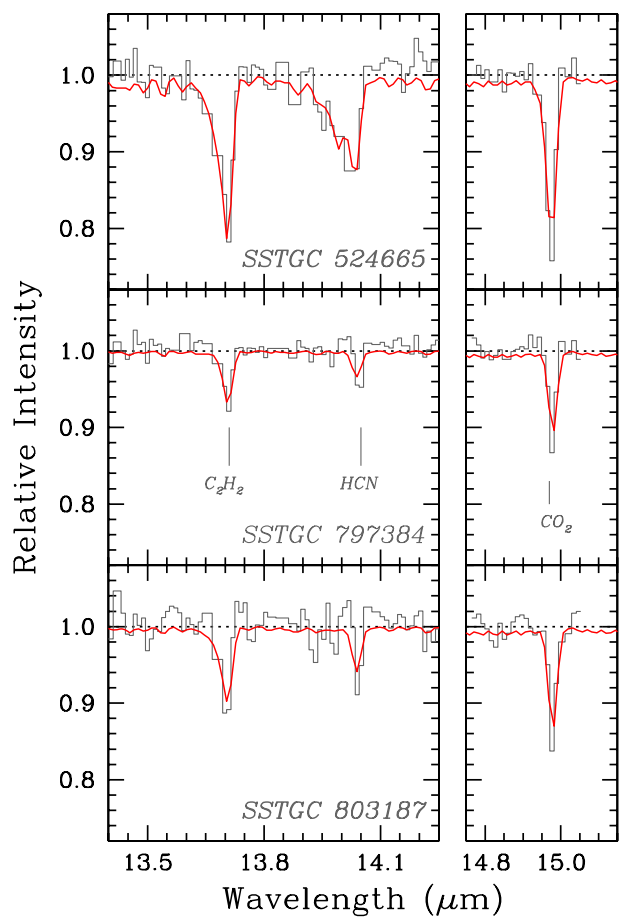

Figure 2. Gas-phase molecular absorptions from $\mathrm{C}_{2} \mathrm{H}_{2} \nu_{5}=1-0(13.71 \mu \mathrm{m})$, $\mathrm{HCN} \nu_{2}=1-0(14.05 \mu \mathrm{m})$, and $\mathrm{CO}_{2} \nu_{2}=1-0(14.97 \mu \mathrm{m})$. Best-fitting models are shown in solid lines.

the excitation temperature $\left(T_{\mathrm{ex}}\right)$ and column density $\left(N_{\mathrm{col}}\right)$ for each molecular species, we used model spectra from Cami et al. (2006) based on HITRAN04 linelist (Rothman et al. 2005) for $\mathrm{C}_{2} \mathrm{H}_{2}$ and $\mathrm{HCN}$, and those based on HITEMP (Rothman et al. 1996) for $\mathrm{CO}_{2}$. A second-order polynomial was used to set a local continuum at $13.30 \mu \mathrm{m} \leqslant \lambda \leqslant 14.55 \mu \mathrm{m}$ for $\mathrm{C}_{2} \mathrm{H}_{2}$ and $\mathrm{HCN}$, and $14.77 \mu \mathrm{m} \leqslant \lambda \leqslant 15.06 \mu \mathrm{m}$ for $\mathrm{CO}_{2}$. We did not include isotopes in the computation because of the limited parameter span in the model grids. However, even a relatively high fraction of isotopes in $\mathrm{GC}\left({ }^{12} \mathrm{C} /{ }^{13} \mathrm{C} \approx 23\right.$; Wannier 1980) has a negligible impact in the model fitting.

We first made a fit to $\mathrm{C}_{2} \mathrm{H}_{2}$, and subtracted its contribution to the absorption near weaker HCN bands. Best-fitting model $T_{\text {ex }}$ and $N_{\text {col }}$ were found by searching the minimum $\chi^{2}$ of the fits over $100 \mathrm{~K} \leqslant T_{\mathrm{ex}} \leqslant 1000 \mathrm{~K}$ in steps of $\Delta T_{\mathrm{ex}}=100 \mathrm{~K}$, and $15 \leqslant \log N_{\text {col }} \leqslant 18$ for $\mathrm{C}_{2} \mathrm{H}_{2}, 16 \leqslant \log N_{\text {col }} \leqslant 18$ for $\mathrm{HCN}$, and $16 \leqslant \log N_{\text {col }} \leqslant 22$ for $\mathrm{CO}_{2}$ with intervals of $0.1 \mathrm{dex}$. The solid lines in Figure 2 show our best-fitting models, and their $T_{\text {ex }}$ and $N_{\text {col }}$ are listed in Table 1. Errors in these parameters were estimated from $\Delta \chi^{2}$, where $1 \sigma$ measurement errors were taken from the scatter of flux in the spectra. Systematic errors from background subtraction and nodding differences were then added in quadrature. We tested with varying covering factors, but found that best-fitting case yields its value equal to or close to unity.

These gaseous bandheads have been detected in absorption toward YSOs, tracing the warm and dense gas in the circumstellar disk and/or envelopes (e.g., Lahuis \& van Dishoeck 2000; Boonman et al. 2003; Knez et al. 2009). They are sometimes detected in the photosphere and/or the circumstellar envelope of carbon-rich asymptotic giant branch stars (e.g., Aoki et al. 1999), but carbon stars have not been found in the GC region (e.g., Guglielmo et al. 1998).

The above estimates are based on models with a Doppler parameter $b=3 \mathrm{~km} \mathrm{~s}^{-1}$. The line width measurements of these molecules for several massive YSOs and that of the strongest $\mathrm{H}_{2} \mathrm{CO}$ absorption components near SSTGC 803187 are in the range of $b=1-7 \mathrm{~km} \mathrm{~s}^{-1}$ (e.g., Mehringer 1995; van der Tak et al. 2000; Knez et al. 2009). There are limited model grids at $b=10 \mathrm{~km} \mathrm{~s}^{-1}$ for $\mathrm{C}_{2} \mathrm{H}_{2}$ and $\mathrm{HCN}$, but $T_{\text {ex }}$ and $N_{\text {col }}$ were generally found within $2 \sigma$ from those at $b=3 \mathrm{~km} \mathrm{~s}^{-1}$.

Figure 3 shows optical depth spectra of our sources (gray) at $\sim 15.2 \mu \mathrm{m}$, where the strong and wide $\mathrm{CO}_{2}$ ice absorption is seen. We set a local continuum over $14.5 \mu \mathrm{m} \leqslant \lambda \leqslant 16.5 \mu \mathrm{m}$ using a third-order polynomial, and followed the prescription in Pontoppidan et al. (2008) to decompose the absorption profile with five laboratory spectral components: polar $\left(\mathrm{CO}_{2}: \mathrm{H}_{2} \mathrm{O}=\right.$ $14: 100$ at $10 \mathrm{~K}$; dotted line, centered at $\sim 15.3 \mu \mathrm{m}$ ), apolar $\left(\mathrm{CO}: \mathrm{CO}_{2}=100: 70\right.$ at $10 \mathrm{~K}$; dotted line, centered at $\sim 15.1 \mu \mathrm{m})$, pure $\mathrm{CO}_{2}(15 \mathrm{~K}$; blue shaded $)$, diluted $\mathrm{CO}_{2}$ $\left(\mathrm{CO}: \mathrm{CO}_{2}=100: 4\right.$ at $10 \mathrm{~K}$; black solid line), and $15.4 \mu \mathrm{m}$ shoulder $\mathrm{CO}_{2}$ ice profile (modeled with two Gaussians in wavenumber space; orange shaded). We found a best-fitting set of models from the nonlinear least-squares fitting routine MPFIT (Markwardt 2009). The green solid line represents the sum of all of the ice components, and the $\mathrm{CO}_{2}$ ice column density in Table 1 was estimated from the integrated absorption, adopting the integrated line strength $A=1.1 \times 10^{-17} \mathrm{~cm}^{\text {molecule }}{ }^{-1}$ (Gerakines et al. 1995).

Unlike the $\mathrm{CO}_{2}$ absorption profiles observed in quiescent molecular clouds (e.g., Whittet et al. 2009), the $15.2 \mu \mathrm{m}$ band in Figure 3 shows a remarkable double-peaked profile. Doublepeaked profiles are commonly observed toward YSOs (e.g., Gerakines et al. 1999; Pontoppidan et al. 2008), and are ascribed to pure $\mathrm{CO}_{2}$ ices resulting from crystallization of heated $\mathrm{H}_{2} \mathrm{O}$-rich ices. However, the double peaks toward the GC candidate YSOs are centered at longer wavelengths $(15.15 \mu \mathrm{m}$ and $15.4 \mu \mathrm{m}$ versus $15.10 \mu \mathrm{m}$ and $15.25 \mu \mathrm{m})$, and result from CO-rich (15.15 $\mu \mathrm{m}$ peak) and $\mathrm{CH}_{3} \mathrm{OH}$-rich ices (15.4 $\mu \mathrm{m}$ peak; see Figure 3).

The strength of the $15.4 \mu \mathrm{m}$ peak is similar to that of the well-studied embedded massive YSO W33A (Gerakines et al. 1999, bottom panel in Figure 3). It is ascribed to a Lewis acid-base interaction of $\mathrm{CO}_{2}$ (the Lewis acid) with $\mathrm{CH}_{3} \mathrm{OH}$ (Dartois et al. 1999a). Other species could be acting as a base as well, but $\mathrm{CH}_{3} \mathrm{OH}$ is preferred due to its high abundance toward W33A: 5\%-22\% relative to solid $\mathrm{H}_{2} \mathrm{O}$ (Dartois et al. 1999b). Two other YSOs (AFGL 7009S, AFGL 2136) show a prominent $15.4 \mu \mathrm{m}$ peak, and indeed these sources have high $\mathrm{CH}_{3} \mathrm{OH}$ abundances as well (Dartois et al. 1999b; Gibb et al. 2004). This suggests that the GC candidate YSOs have high solid $\mathrm{CH}_{3} \mathrm{OH}$ abundances as well. ${ }^{13}$ Although the origin of the large quantities of $\mathrm{CH}_{3} \mathrm{OH}$ in the previously studied massive YSOs is not fully understood (Dartois et al. 1999a), so far all lines of sight with high solid $\mathrm{CH}_{3} \mathrm{OH}$ abundances are associated with star formation, strengthening the idea that the sources studied in this Letter are indeed YSOs.

To derive abundances of these molecular absorptions with respect to the hydrogen and solid $\mathrm{H}_{2} \mathrm{O}$ column densities, we followed the procedure in Boogert et al. (2008) to fit the $\mathrm{H}_{2} \mathrm{O}$ ice and silicate absorption profiles to SL and LL spectra. Figure 4 shows an example for SSTGC 797384. We used the silicate absorption profiles in the line of sight to the GC (GCS 3 spectrum; Kemper et al. 2004) plus a laboratory spectrum of pure amorphous $\mathrm{H}_{2} \mathrm{O}$ ice at $T=10 \mathrm{~K}$ (Hudgins et al.

\footnotetext{
13 This needs to be verified by independent $L$-band spectroscopy of the
} $3.53 \mu \mathrm{m} \mathrm{C}-\mathrm{H}$ stretch mode of $\mathrm{CH}_{3} \mathrm{OH}$ (e.g., Dartois et al. 1999b). 
Table 1

Properties of the Sample

\begin{tabular}{|c|c|c|c|c|}
\hline Quantities & Units & SSTGC 524665 & SSTGC 797384 & SSTGC 803187 \\
\hline R.A.(J2000.0) & $\mathrm{h}: \mathrm{m}: \mathrm{s}$ & $17: 45: 39.86$ & $17: 47: 23.68$ & $17: 47: 26.29$ \\
\hline Decl.(J2000.0) & $\mathrm{d}: \mathrm{m}: \mathrm{s}$ & $-29: 23: 23.4$ & $-28: 23: 34.6$ & $-28: 22: 1.5$ \\
\hline UKIDSS $J^{\mathrm{a}}$ & mag & $\ldots$ & $18.23 \pm 0.06$ & $17.39 \pm 0.03$ \\
\hline UKIDSS $H^{\mathrm{a}}$ & mag & $\ldots$ & $14.68 \pm 0.01$ & $16.60 \pm 0.05$ \\
\hline UKIDSS $K^{\mathrm{a}}$ & mag & $15.71 \pm 0.10$ & $12.92 \pm 0.01$ & $14.37 \pm 0.02$ \\
\hline $\operatorname{IRAC}[3.6]^{\mathrm{b}}$ & mag & $11.42 \pm 0.01$ & $\ldots$ & $12.22 \pm 0.02$ \\
\hline $\operatorname{IRAC}[4.5]^{\mathrm{b}}$ & mag & $8.63 \pm 0.01$ & $9.41 \pm 0.01$ & $8.97 \pm 0.01$ \\
\hline IRAC $[5.8]^{\mathrm{b}}$ & mag & $7.08 \pm 0.01$ & $7.66 \pm 0.01$ & $7.24 \pm 0.01$ \\
\hline $\operatorname{IRAC}[8.0]^{\mathrm{b}}$ & mag & $6.13 \pm 0.01$ & $5.64 \pm 0.01$ & $5.11 \pm 0.01$ \\
\hline MIPS $[24]^{\mathrm{c}}$ & mag & $1.54 \pm 0.01$ & $0.55 \pm 0.01$ & $\ldots$ \\
\hline$T_{\mathrm{ex}}\left(\mathrm{C}_{2} \mathrm{H}_{2}\right.$ gas $)$ & $\mathrm{K}$ & $300 \pm 150$ & $200 \pm 150$ & $300 \pm 150$ \\
\hline$T_{\mathrm{ex}}(\mathrm{HCNgas})$ & $\mathrm{K}$ & $400 \pm 50$ & $100 \pm 50$ & $100 \pm 50$ \\
\hline$T_{\mathrm{ex}}\left(\mathrm{CO}_{2} \mathrm{gas}\right)$ & $\mathrm{K}$ & $200 \pm 50$ & $100 \pm 50$ & $100 \pm 50$ \\
\hline$N_{\mathrm{col}}\left(\mathrm{C}_{2} \mathrm{H}_{2}\right.$ gas $)$ & $10^{16} \mathrm{~cm}^{-2}$ & $7.9 \pm 3.3$ & $1.0 \pm 0.3$ & $2.0 \pm 0.8$ \\
\hline$N_{\text {col }}$ (HCNgas) & $10^{16} \mathrm{~cm}^{-2}$ & $15.8 \pm 4.6$ & $1.0 \pm 0.4$ & $2.0 \pm 0.9$ \\
\hline$N_{\text {col }}\left(\mathrm{CO}_{2}\right.$ gas $)$ & $10^{16} \mathrm{~cm}^{-2}$ & $20.0 \pm 5.6$ & $5.0 \pm 1.5$ & $7.9 \pm 2.8$ \\
\hline$N_{\text {col }}\left(\mathrm{CO}_{2}\right.$ solid $)$ & $10^{19} \mathrm{~cm}^{-2}$ & $0.11 \pm 0.01$ & $0.13 \pm 0.01$ & $0.21 \pm 0.01$ \\
\hline$N_{\text {col }}\left(\mathrm{H}_{2}\right.$ Osolid, $\left.6 \mu \mathrm{m}\right)$ & $10^{19} \mathrm{~cm}^{-2}$ & $<1.7$ & $<2.3$ & $<4.7$ \\
\hline$N_{\text {col }}\left(\mathrm{H}_{2}\right.$ Osolid, $\left.13 \mu \mathrm{m}\right)$ & $10^{19} \mathrm{~cm}^{-2}$ & $0.6 \pm 0.4$ & $1.3 \pm 0.4$ & $1.9 \pm 0.3$ \\
\hline$N_{\mathrm{col}}\left(\mathrm{H}_{2}\right)$ & $10^{22} \mathrm{~cm}^{-2}$ & $2.3 \pm 0.2$ & $5.0 \pm 0.5$ & $5.8 \pm 0.7$ \\
\hline$N_{\mathrm{C}_{2} \mathrm{H}_{2}} / N_{\mathrm{H}_{2}}$ & $10^{-7}$ & $34.3 \pm 14.7$ & $2.0 \pm 0.6$ & $3.4 \pm 1.4$ \\
\hline$N_{\mathrm{HCN}} / N_{\mathrm{H}_{2}}$ & $10^{-7}$ & $68.7 \pm 20.9$ & $2.0 \pm 0.8$ & $3.4 \pm 1.6$ \\
\hline$N_{\mathrm{CO}_{2}, \text { gas }} / N_{\mathrm{H}_{2}}$ & $10^{-7}$ & $87.0 \pm 25.5$ & $10.0 \pm 3.2$ & $13.6 \pm 5.1$ \\
\hline$N_{\mathrm{CO}_{2}, \text { gas }} / N_{\mathrm{CO}_{2}, \text { solid }}$ & & $0.18 \pm 0.05$ & $0.04 \pm 0.01$ & $0.04 \pm 0.01$ \\
\hline$N_{\mathrm{CO}_{2}, \text { solid }} / N_{\mathrm{H}_{2} \mathrm{O}, \text { solid }}$ & & $0.18 \pm 0.12$ & $0.10 \pm 0.03$ & $0.11 \pm 0.02$ \\
\hline$A_{V}\left(\tau_{9.7 \mu m}\right)$ & mag & $27 \pm 3$ & $53 \pm 5$ & $62 \pm 7$ \\
\hline$A_{V}(\text { color })^{\mathrm{d}}$ & mag & $29.0 \pm 2.6$ & $32.0 \pm 2.6$ & $27.0 \pm 5.4$ \\
\hline
\end{tabular}

Notes. Random errors are shown in the photometry.

a Aperture3 magnitudes from UKIDSS DR2 (Warren et al. 2007).

${ }^{b}$ Photometry from Ramírez et al. (2008).

${ }^{\mathrm{c}}$ Photometry from MIPSGAL (S. Carey 2008, private communication).

d Based on the 2MASS and IRAC color-magnitude diagrams of GC red giant branch stars within 2' of the source (Schultheis et al. 2009).

1993). We simultaneously fit a second-order polynomial for a pseudo-continuum (i.e., including corrections for the continuous extinction), the silicate profile, and $\mathrm{H}_{2} \mathrm{O}$ ice absorption to the $5 \mu \mathrm{m} \leqslant \lambda \leqslant 32 \mu \mathrm{m}$ spectrum. We masked absorption features at $6 \mu \mathrm{m}, 7 \mu \mathrm{m}$, and $15 \mu \mathrm{m}$, and all unresolved emission lines, before performing a nonlinear least-squares fit.

Best-fitting parameters are listed in Table 1. We obtained a total hydrogen column density from the optical depth of the $9.7 \mu \mathrm{m}$ silicate absorption, assuming $A_{V} / \tau_{9.7}=9$ (Roche \& Aitken 1985) and $N_{\mathrm{H}} / A_{V} \approx 1.87 \times 10^{21} \mathrm{~cm}^{-2} \mathrm{mag}^{-1}$ (Bohlin et al. 1978) at $R_{V}=3.1$. The $\mathrm{H}_{2}$ column density was then approximated by $N_{\mathrm{H}_{2}}=N_{\mathrm{H}} / 2$. The ice column density for the $13 \mu \mathrm{m}$ librational $\mathrm{H}_{2} \mathrm{O}$ absorption was estimated from the integrated absorption of the best-fitting $\mathrm{H}_{2} \mathrm{O}$ model. The $\mathrm{H}_{2} \mathrm{O}$ ice column density from the $6 \mu \mathrm{m}$ bending mode, fit separately after fixing the continuum and extinction to previously found values, is an upper limit because the $6 \mu \mathrm{m}$ absorption is not solely due to $\mathrm{H}_{2} \mathrm{O}$ ice. We adopted the integrated line strengths $A=1.2 \times 10^{-17} \mathrm{~cm}$ molecule ${ }^{-1}$ for the bending mode and $A=3.1 \times 10^{-17} \mathrm{~cm}$ molecule ${ }^{-1}$ for the librational mode (Gerakines et al. 1995). Errors in these parameters (Table 1) are formal estimates made by varying the range of wavelengths that we used for the $9.7 \mu \mathrm{m}$ silicate fitting, or by taking a few different ways of setting the continuum.

The gas-phase molecular abundances relative to $\mathrm{H}_{2}$ are listed in Table 1. Our derived abundances of $\sim 10^{-7}-10^{-6}$ for $\mathrm{C}_{2} \mathrm{H}_{2}$ and $\mathrm{HCN}$ are comparable to those found for massive YSOs (Lahuis \& van Dishoeck 2000; Knez et al. 2009), although abundances for SSTGC 524665 have large errors. Intervening molecular clouds in the line of sight to the GC are less likely the main cause of these absorptions, because the average HCN abundance of $2.5 \times 10^{-8}$ (Greaves \& Nyman 1996) toward Sgr $\mathrm{B} 2(\mathrm{M})$ is an order of magnitude lower than our measurements. Our gas-phase $\mathrm{CO}_{2}$ abundances are an order of magnitude larger than those found toward massive YSOs in Boonman et al. (2003), but our gas to solid abundance ratios for $\mathrm{CO}_{2}$ are consistent with their estimates $\left(10^{-1}-10^{-2}\right)$. Our abundance of $\mathrm{CO}_{2}$ ice relative to $\mathrm{H}_{2} \mathrm{O}$ ice is within the range $(0.10-0.23)$ found toward massive YSOs (Gerakines et al. 1999).

Finally, Table 1 lists our estimates on $A_{V}$ from the $9.7 \mu \mathrm{m}$ silicate absorption and those from Schultheis et al. (2009), based on the 2MASS and IRAC color-magnitude diagrams of GC red giant branch stars within $2^{\prime}$ of the source. Both SSTGC 797384 and SSTGC 803187 have higher $A_{V}$ values than the average for field stars, implying that a significant fraction of the attenuation is intrinsic to the source. SSTGC 524665 has a lower $A_{V}$, comparable to the average value for surrounding field stars. We also note that SSTGC 524665 is located at $b \approx-0.2$, so it is possible that it is in front of the GC. If we assume a distance of $8 \mathrm{kpc}$ for all three sources, and adopt the extinction of surrounding field stars as the foreground extinction to each source, then we derive stellar masses of $12 \pm 3 M_{\odot}, 14 \pm 3 M_{\odot}$, and $17 \pm 6 M_{\odot}$ for SSTGC 524665, SSTGC 797384, and SSTGC 803187 , respectively, by using a grid of YSO models (Robitaille et al. 2006, 2007). More detailed discussion of the model fitting will presented in a future paper. 


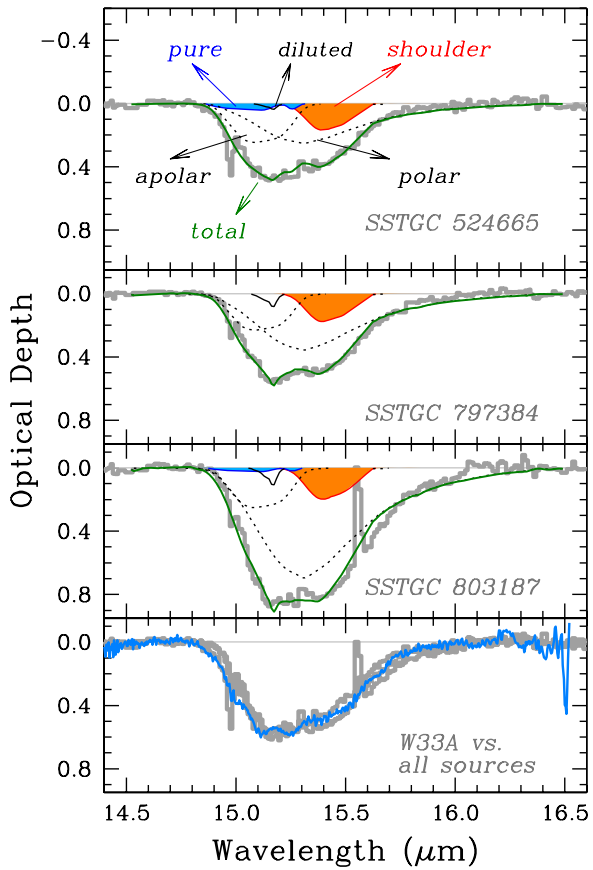

Figure 3. Optical depth spectra of solid-phase absorption from the $\mathrm{CO}_{2}$ ice bending mode. Best-fitting $\mathrm{CO}_{2}$ ice models and individual $\mathrm{CO}_{2}$ ice components are shown for each target: polar (dotted line, centered at $\sim 15.3 \mu \mathrm{m}$ ), apolar (dotted line, centered at $\sim 15.1 \mu \mathrm{m}$ ), pure (blue shaded), diluted (black solid line), $15.4 \mu \mathrm{m}$ shoulder (orange-shaded), and the sum of these absorption components (green line). The bottom panel shows a comparison of the ice absorption profile between our sources (gray) and massive YSO W33A (blue). The optical depths for our targets were scaled in the bottom panel for comparison.

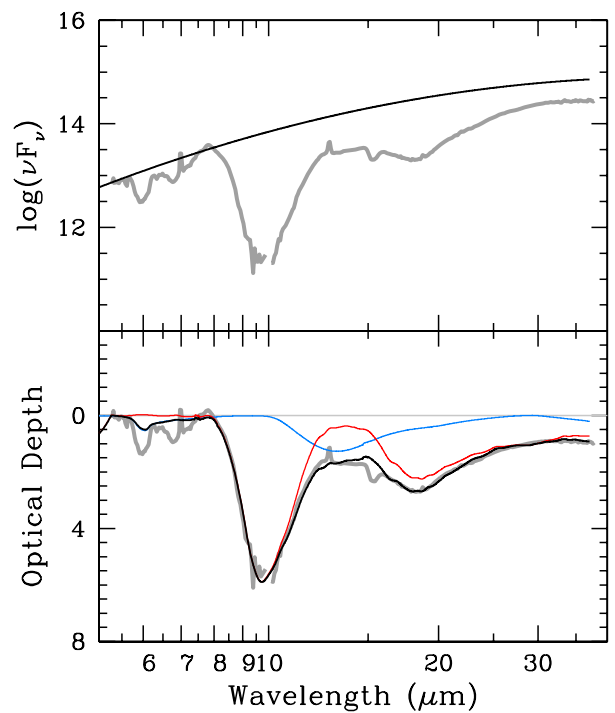

Figure 4. Fit to the $\mathrm{H}_{2} \mathrm{O}$ ice and silicate absorption for SSTGC 797384. Top: SL and LL data (gray), with a best-fitting pseudo-continuum (black line). Bottom: decomposition of optical depth spectra (gray) with the silicate (red) and the laboratory $\mathrm{H}_{2} \mathrm{O}$ ice profiles (blue). Black line represents a sum of these two components.

To summarize, we presented the evidence from IRS spectra for the first spectroscopic identification of massive YSOs in the GC. In our next paper (D. An 2009, in preparation), we will present the results for all 107 YSO candidates, together with additional data from millimeter to radio observations, and use them to better understand the nature of these embedded sources.

We thank David Ardila for helpful discussions of the IRS data reduction. We thank Sean Carey for providing us MIPS photometry before publication. D.A. and S.R. thank John Stauffer for helpful discussions. This work is based on observations made with the Spitzer Space Telescope, which is operated by the Jet Propulsion Laboratory, California Institute of Technology under a contract with NASA. Support for this work was provided by NASA through an award issued by JPL/Caltech. This research has made use of the SIMBAD database, operated at CDS, Strasbourg, France.

\section{REFERENCES}

Aoki, W., Tsuji, T., \& Ohnaka, K. 1999, A\&A, 350, 945

Bohlin, R. C., Savage, B. D., \& Drake, J. F. 1978, ApJ, 224, 132

Boogert, A. C. A., et al. 2008, ApJ, 678, 985

Boonman, A. M. S., van Dishoeck, E. F., Lahuis, F., \& Doty, S. D. 2003, A\&A, 399, 1063

Cami, J., Markwick-Kemper, A. J., \& Van Malderen, R. 2009, ApJS, submitted Carey, S. J., et al. 2009, PASP, 121, 76

Dartois, E., Demyk, K., d'Hendecourt, L., \& Ehrenfreund, P. 1999a, A\&A, 351, 1066

Dartois, E., Schutte, W., Geballe, T. R., Demyk, K., Ehrenfreund, P., \& D’Hendecourt, L. 1999b, A\&A, 342, L32

Fazio, G. G., et al. 2004, ApJS, 154, 10

Felli, M., Testi, L., Schuller, F., \& Omont, A. 2002, A\&A, 392, 971

Forster, J. R., \& Caswell, J. L. 1989, A\&A, 213, 339

Gerakines, P. A., Schutte, W. A., Greenberg, J. M., \& van Dishoeck, E. F. 1995 A\&A, 296, 810

Gerakines, P. A., et al. 1999, ApJ, 522, 357

Gibb, E. L., Whittet, D. C. B., Boogert, A. C. A., \& Tielens, A. G. G. M. 2004, ApJS, 151, 35

Greaves, J. S., \& Nyman, L.-A. 1996, A\&A, 305, 950

Guglielmo, F., Le Bertre, T., \& Epchtein, N. 1998, A\&A, 334, 609

Houck, J. R., et al. 2004, ApJS, 154, 18

Hudgins, D. M., Sandford, S. A., Allamandola, L. J., \& Tielens, A. G. G. M. 1993, ApJS, 86, 713

Kemper, F., Vriend, W. J., \& Tielens, A. G. G. M. 2004, ApJ, 609, 826

Knez, C., Lacy, J. H., Evans, N. J., van Dishoeck, E. F., \& Richter, M. J. 2009, ApJ, 696, 471

Lahuis, F., \& van Dishoeck, E. F. 2000, A\&A, 355, 699

Markwardt, C. B. 2009, arXiv:0902.2850

Mehringer, D. M. 1995, ApJ, 454, 782

Mehringer, D. M., Palmer, P., Goss, W. M., \& Yusef-Zadeh, F. 1993, ApJ, 412, 684

Morris, M., \& Serabyn, E. 1996, ARA\&A, 34, 645

Omont, A., et al. 2003, A\&A, 403, 975

Pontoppidan, K. M., et al. 2008, ApJ, 678, 1005

Ramírez, S. V., Arendt, R. G., Sellgren, K., Stolovy, S. R., Cotera, A., Smith, H. A., \& Yusef-Zadeh, F. 2008, ApJS, 175, 147

Robitaille, T. P., Whitney, B. A., Indebetouw, R., \& Wood, K. 2007, ApJS, 169, 328

Robitaille, T. P., Whitney, B. A., Indebetouw, R., Wood, K., \& Denzmore, P. 2006, ApJS, 167, 256

Roche, P. F., \& Aitken, D. K. 1985, MNRAS, 215, 425

Rothman, L. S., et al. 1996, J. Quant. Spectrosc. Radiat. Transfer, 60, 665

Rothman, L. S., et al. 2005, J. Quant. Spectrosc. Radiat. Transfer, 96, 139

Schuller, F., Omont, A., Glass, I. S., Schultheis, M., Egan, M. P., \& Price, S. D. 2006, A\&A, 453, 535

Schultheis, M., Lançon, A., Omont, A., Schuller, F., \& Ojha, D. K. 2003, A\&A, 405, 531

Schultheis, M., Sellgren, K., Ramírez, S., Stolovy, S., Ganesh, S., Glass, I. S., \& Girardi, L. 2009, A\&A, 495, 157

Skrutskie, M. F., et al. 2006, AJ, 131, 1163

Smith, H. A., Hora, J. L., Marengo, M., \& Pipher, J. L. 2006, ApJ, 645, 1264

Stolovy, S., et al. 2006, J. Phys. Conf. Ser., 54, 176

van der Tak, F. F. S., van Dishoeck, E. F., Evans, N. J., II, \& Blake, G. A 2000, ApJ, 537, 283

Wannier, P. G. 1980, ARA\&A, 18, 399

Warren, S. J., et al. 2007, arXiv:astro-ph/0703037

Werner, M. W., et al. 2004, ApJS, 154, 1

Whitney, B. A., Indebetouw, R., Bjorkman, J. E., \& Wood, K. 2004, ApJ, 617, 1177

Whitney, B. A., Wood, K., Bjorkman, J. E., \& Cohen, M. 2003, ApJ, 598, 1079

Whittet, D. C. B., Cook, A. M., Chiar, J. E., Pendleton, Y. J., Shenoy, S. S., \& Gerakines, P. A. 2009, ApJ, 695, 94

Yusef-Zadeh, F., et al. 2009, arXiv:0905.2161 